\title{
Asociatividad y desarrollo económico de los productores de cacao en la provincia de Los Ríos
}

\section{Associativity and economic development of cocoa producers in the province of Los Ríos}

\author{
Angel Eduardo González Vásquez ${ }^{1 *}$ \\ ${ }^{1}$ Universidad Politécnica Salesiana \\ *agonzalez@ups.edu.ec
}

DOI: https://doi.org/10.26871/killkana_social.v2i4.369

\begin{abstract}
Resumen
La efectividad de la asociatividad se mide en el bienestar económico y social que otorga a sus practicantes. La estrategia de asociarse en el sector cacaotero debe ser medida y evaluada para encontrar oportunidades de mejoras. Con esta perspectiva, el presente artículo tiene como objetivo determinar si la asociatividad influye significativamente en el desarrollo económico de los productores de cacao en la provincia de Los Ríos del Ecuador. Se analiza la tenencia de la tierra, inestabilidad de los precios, bonanza del cacao, estrategias de cultivos combinados y exigencias de certificaciones como antecedentes a la problemática del productor cacaotero asociado. La investigación es de tipo cuantitativa, aplicada, no experimental y transeccional correlacional. Para cumplir con el objetivo se contrasta estadísticamente la hipótesis de investigación. Los resultados demuestran una influencia significativa.
\end{abstract}

Palabras clave: asociatividad, asociación, cacao, desarrollo económico, estrategia.

\begin{abstract}
The effectiveness of associativity is measured in the economic and social welfare that it gives to its practitioners. The strategy of partnering in the cocoa sector must be measured and evaluated in order to find opportunities for improvement. With this perspective, the objective of this article is to determine if associativity significantly influences the economic development of cocoa producers in the province of Los Rios of Ecuador. We analyze land tenure, price instability, cocoa bonanza, combined crop strategies and certification requirements as antecedents to the problematic of the associated cocoa producer. The research is quantitative, applied, non-experimental and transectional correlational. To achieve the objective, the research hypothesis is statistically contrasted. The results show a significant influence.
\end{abstract}

Key words: associativity, association, cocoa, economic development, strategy.

\section{Introducción}

Cuán importante es utilizar la estrategia de asociarse en el sector de la agricultura para lograr mejorar los ingresos económicos, sin embargo, en ocasiones se necesita conocer el verdadero impacto que tiene la asociatividad en las aspiraciones de bienestar de los agricultores. La efectividad de la asociatividad, en los aspectos económicos, se mide por el beneficio que otorga la práctica de la misma en el incremento de utilidades y, por consiguiente, en la mejora de la calidad de vida. Por tanto, en esta investigación se pretende medir la contribución que presta utilizar la estrategia de asociarse en el aumento de los ingresos.

Se tomó como población objeto de estudio a los agricultores que producen cacao y, además, que pertenecen a una asociación agrícola que tiene como actividad principal la comercialización de cacao. Se delimitó el estudio sola- mente a la provincia de los Ríos del Ecuador. La razón de escoger al sector cacaotero, es porque la evolución en el comercio del cacao a nivel nacional e internacional tiene una tendencia y posiblemente obligación hacia la asociatividad.

Es necesario revisar la historia de lo que pasó en el país con respecto a la tenencia de la tierra, porque se convirtió en la coyuntura actual del sector cacaotero. Es así que, en el Ecuador, se establecieron algunos modelos de desarrollo amparados en leyes en procura de mejorar las condiciones económicas y sociales de los sectores rurales. Se ejecutó la Reforma Agraria en el año 1964, un programa amparado en la ley que redistribuía la tenencia de la tierra. Con este modelo de desarrollo, se obtuvo que grandes extensiones de terrenos pasen a ser productivas, haciendas de gran tamaño se parcelaron y se las entregaron a agricultores para que las trabajen. Por lo general, eran terrenos baldíos cuyos 
propietarios no tenían la capacidad de laborar en ellos, en otras ocasiones, se irrespetó el derecho a la propiedad privada, por cuanto unas haciendas que estaban en producción fueron invadidas, con posesiones de hecho y a la fuerza.

Del resultado de la Reforma Agraria, se logró el aumento de productos agrícolas, se incrementó el número de consumidores con mayor poder adquisitivo, al aumentar el empleo en las áreas rurales. Así también, de manera general, la presión social por la tenencia de la tierra disminuyó. Sin embargo, surgieron problemas por los abusos por parte de algunos campesinos, en ocasiones, en ciertos sectores del país, en vez de disminuir la ansiedad por el deseo de poseer terrenos, más se incrementaba las aspiraciones y se tornaban en luchas sociales, terminando en conflictos armados. Posteriormente, en 1994, bajo el enfoque o corriente del neoliberalismo, se promulgó la "Ley de Desarrollo Agrario", una nueva ley con un enfoque práctico que garantizaba los emprendimientos en el sector rural; entre las intenciones, lo resume Brassel, Herrera, y Laforge (2008), "promociona el mercado de tierras; elimina todas las restricciones posibles a la transferencia de las propiedades rústicas; la mediana y gran propiedad tienen garantía del Estado; autoriza el fraccionamiento de tierras comunales y su transferencia a terceros, vía mercado" (p. 20).

En el 2017, se promulga la "Ley Orgánica de Tierras Rurales y Territorios Ancestrales", con el objetivo de plasmar garantías sobre los alimentos que comen los ecuatorianos ancestralmente (soberanía alimentaria), proteger ecosistemas y aumentar la producción. Uno de los puntos discutidos por los grupos empresariales, es el que establece: "Prohibición del latifundio y de la concentración de la tierra rural. El Estado hará efectiva la prohibición del latifundio e impedirá la concentración de la tierra rural" (Asamblea Nacional, 2016, art. 7).

En esta coyuntura, lo que se observa en el 2018 en la provincia de los Ríos es a muchos agricultores con parcelas pequeñas menores de 10 hectáreas. La tendencia a la fragmentación ha de seguir, porque las personas mueren y heredan a sus hijos, lo que de alguna manera ha ido fraccionando las haciendas producto del reparto de la herencia. También se da, que aparecen grupos empresariales que compran los predios heredados para el cultivo de plantaciones de palma africana, banano o el mismo cacao a gran escala y solo para la exportación. Cuando sucede esto, se vuelve otra vez a la problemática, el agricultor vive en su casa en un recinto o caserío, ya no tiene tierras y trabaja para las empresas agrícolas por un sueldo hasta cuando la edad y salud le permita laborar. La jubilación en el sector de la agricultura tiene muchas falencias aún, el seguro campesino no satisface las necesidades de una persona adulta mayor.

En este contexto, también surgió el asociacionismo (tendencia a crear asociaciones), los campesinos se agrupaban y formaron asociaciones de agricultores de diferente índole, hay actualmente, asociaciones de arroceros, bana- neros, maiceros, palma africana, cacao, entre otros. Otros grupos se denominan simplemente asociaciones agrícolas o agropecuarias, es decir, tienen un campo de acción amplio. Centrándose a la presente investigación, las asociaciones que tienen como finalidad o actividad principal el acopio y comercialización de cacao, han evidenciado logros más allá de lo económico, pues han logrado mejorar en los aspectos sociales y en la educación para mitigar el impacto al medio ambiente ocasionada por la producción de cacao.

Actualmente, los agricultores por su propia experiencia, no se fían de la bonanza de los productos agrícolas. Los precios varían y en ocasiones caen a un valor menor a los costos de producción. Lo penoso de esto es que las inversiones en la agricultura tienen su tiempo y dedicación $\mathrm{y}$, en un momento, simplemente el cultivo ya no es rentable. Por otra parte, la ventaja de los terrenos de la provincia de Los Ríos es que son muy versátiles, los campesinos pasan de cultivos de ciclo corto (cultivos con un tiempo de producción menor a seis meses, como el maíz, soya, sandías, tomates, entre otros) a cultivos que necesitan más tiempo para poder cosechar como el cacao, banano, café o palma africana. También existen suelos que solo presentan cualidades para un determinado producto, como sucede en las cuencas de los ríos, en estos sectores, es preferible solo sembrar arroz.

En este entorno económico, los propietarios de pequeñas fincas optaron por mantener cultivos combinados, o sea, que no se cultiva intensivamente un solo producto. Se cultiva maracuyá, papaya, plátano, café, cítricos, cacao, entre otros, respetando los espacios vitales para el crecimiento y desarrollo de cada planta o cultivo. Se puede evidenciar, en muchos casos, haciendas pequeñas de menos de 5 hectáreas con una eficiencia alta en el uso de los suelos. No obstante, que se utilice el $100 \%$ del terreno, no significa que sea eficaz y rentable el trabajo realizado. Es en estas pequeñas parcelas donde el cacao surge como una alternativa de trabajar con eficacia (producir las cosas correctas que se vendan a buen precio sin dañar la soberanía alimentaria de cada nucleo familiar). De estos productos, el cacao ha mantenido desde el año 2000 un precio atractivo que motivó la inversión por parte de los agricultores y que les sirve aún como una fuente segura de ingresos. Es un fruto no perecible a corto plazo como los cítricos, papayas $\mathrm{u}$ otros, puede inclusive permanecer años, sin perder sus características organolépticas, si está bien conservado. A diferencia de otros productos que necesitarían ser procesados, enlatados o conservados en frío, la pepa de cacao, una vez fermentada y secada, otorga al agricultor una holgura de tiempo para su comercialización. Para entender esta problemática, se ha observado que los agricultores cuando cosechan sus plátanos o naranjas, tienen solamente una o máximo dos semanas para comercializar los mismos, se maduran y pueden perder todo, así que lo rematan al mejor postor.

Pero, cultivar cacao intensivamente también tiene sus riesgos, el cacao es un producto que responde a la ley de 
la oferta y la demanda (sube la oferta, baja el precio); la historia ecuatoriana presenta auges y declives en el precio del cacao. Un peligro latente es que exista una baja de precios por el aumento de la oferta de los países africanos. Si se fomenta aún más el cultivo en África, será dificil mantener los buenos precios que se han venido pagando a nivel internacional. El precio referencial al cierre de octubre de 2018 es de USD 2195 la tonelada (Asociación Nacional de Exportadores de Cacao - Ecuador, 2018). Para comprender este precio, se explica que en el mercado externo se paga a $\$ 2,19$ el kilogramo de cacao. Sin embargo, al agricultor se le paga un valor menor, existe una cadena de intermediación que cobra por su trabajo. No es criticable la intermediación, es inclusive necesaria, sin embargo, en ocasiones se castiga demasiado con el precio, se ha observado que llega hasta un $50 \%$ del valor internacional. Es decir, solo pagan un valor de \$1,085 por kilogramo (datos observados en sitios de compra). Entre las objeciones para pagar un buen precio está la calidad y no poder hacer la trazabilidad, es decir que no se sabe la procedencia y forma de producir (cosechar, fermentar, secar y almacenar) el cacao.

La trazabilidad es definida como: "Posibilidad de identificar el origen y las diferentes etapas de un proceso de producción y distribución de bienes de consumo" (Real Academia Española, 2018). La exigencia de rastrear el origen aumenta cada vez más en los mercados nacionales y extranjeros, se exige que los productos cumplan con las normas, como son la no presencia de químicos nocivos, residuos de animales o ciertos patógenos. Si existe presencia de inconformidades, es conveniente saber en donde ocurrió la contaminación, para que se descarte el lote, más no se castigue a todos los demás. Se le tiene que dar seguimiento o realizar la trazabilidad, para hacer los correctivos. Aunque esto sea una barrera para la comercialización externa (no hay por qué quejarse, es obvio que cada país precautele la salud de la población), en el mercado interno ecuatoriano es más permisible. No obstante, esta exigencia de la trazabilidad fortalece la asociatividad. Para obtener buenos precios, se tiene que conocer el origen y forma de producir los mismos. La mayoría de las asociaciones, a través de su dirigencia, realizan capacitaciones sobre estos temas e inspeccionan las fincas. El factor clave como proveedor de confianza lo puede ofrecer las asociaciones.

Algunas asociaciones de agricultores cacaoteros logran tener el "Sello de Certificación de Comercio Justo FAIRTRADE" para el cacao. Una asociación que logra estas certificaciones publicita que está haciendo las cosas bien, esto es, la dirigencia es competente en el servicio a sus socios. En este aspecto, afirma la Coordinadora Ecuatoriana de Comercio Justo (2018), que lo importante es mejorar las relaciones comerciales y el poder de negociación entre grandes empresarios y pequeños productores, si se puede, disminuir la intermediación, así también, disminuir los riesgos por la inestabilidad de los precios en los mercados. Aunque han existido críticas, en el sentido de "qué tan justo es el Comercio Justo", porque el agricultor asociado no percibe que progresa, lo importante es que las empresas asociativas cacaoteras mantienen relaciones comerciales que fijan buenos precios para el cacao, por consiguiente para sus socios. De igual forma, en los mercados externos se exigen los sellos de certificación ecológica, en este punto, existen quejas por parte de los productores, quienes indican que el trabajo por obtener un cacao tipificado orgánico no es proporcional al dinero recibido.

Con lo expresado, lo que se necesita es medir y evaluar si existe influencia o relación que implique que a más asociatividad habrá más desarrollo económico o que a menos asociatividad habrá menos desarrollo económico. Puede ser que cambiado los contextos o entornos económicos, no exista correlación ni influencia significativa o, también, que exista solo una correlación espuria.

Ante lo expuesto, se formuló el siguiente problema de investigación: ¿La asociatividad influye significativamente en el desarrollo económico de los productores de cacao en la provincia de Los Ríos del Ecuador?

Consecuentemente, se estableció el siguiente objetivo: Determinar si la asociatividad influye significativamente en el desarrollo económico de los productores de cacao en la provincia de Los Ríos del Ecuador.

\section{Marco teórico}

Asociatividad: no está definida aún esta palabra por la Real Academia Española, sin embargo, este concepto ha sido aceptado y ha ido evolucionando su definición por las instituciones de algunos países, también se puede decir, que es un concepto que tiene muchas cosmovisiones, esto es, cómo entienden a la asociatividad las personas según el mundo en que viven. Es conveniente revisar algunas definiciones.

"Se entenderá por asociatividad a la organización voluntaria y no remunerada de individuos o grupos que establecen un vínculo explícito, con el fin de conseguir un objetivo común" (Programa de las Naciones Unidas para el Desarrollo, 2000, p. 116). Esta es una definición de tipo general, tanto para personas o empresas, indica que se persiguen fines comunes y no necesariamente es para obtener lucro o ganancia. Para la Cámara de Comercio de Bogotá (2010), "asociatividad es la unión de voluntades, iniciativas y recursos por parte de un grupo de empresas, alrededor de objetivos comunes, desarrollando un proceso que exige compromiso, persistencia y disciplina" (p. 3). Esta es una definición que tiene una visión desde el punto de vista empresarial, en los negocios se presenta muchas formas de asociarse para emprender, es decir, mantener acuerdos comerciales manteniendo la independencia, ya sea en relaciones verticales y horizontales. Existen las redes de cooperación, joint venture, consorcios, clusters, alianzas en cadenas productivas, entre otros. Las asociaciones cacaoteras se tipificarían como alianzas en cadenas productivas. Para otras personas, en este concepto siempre tiene que recalcarse que se mantiene la independencia, es decir que en cualquier momento se puede salir de la asociación. 
En este caso, el compromiso es solo hasta cuando exista el beneficio, si no hay interés económico, se acaba el compromiso.

Por otra parte, para Borja, Alarcón y Quizhpe (2017), la asociatividad según la cosmovisión indígena es la de trabajo comunal, es ayuda mutua y cooperación para lograr un fin determinado. Bajo esta perspectiva, la comunidad se hace presente para realizar las mingas de limpieza, construir la casa comunal, prestar colaboración en la siembra, entre otros. En estas comunidades, la solidaridad y trabajo en equipo posiblemente estuvo presente mucho antes que los principios de asociatividad se difundan.

Ha existido muchas capacitaciones sobre el tema asociatividad hacia los agricultores desde 1998, gracias a las organizaciones no gubernamentales, instituciones estatales y partes interesadas en fortalecer al sector cacaotero. Se espera ya en el 2018, que el agricultor ecuatoriano, tenga claro los beneficios que le puede aportar.

Las asociaciones de productores de cacao: en el 2018, para el Estado ecuatoriano tienen el nombre de corporaciones, con el objetivo de tipificarlas, establecer indicadores y hacer las estadísticas. En el sector cacaotero existen corporaciones de primer, segundo y tercer grado, cada una con sus características propias. Las asociaciones analizadas en esta investigación son las corporaciones de primer grado. En este sentido, se entiende que: " 1 . Corporaciones de primer grado: son aquellas que agrupan a personas naturales con un fin delimitado, tales como: asociaciones, clubes, comités, colegios profesionales y centros" (Decreto Nº193, 2017, art. 4).

Estas corporaciones, también pueden ampararse en las leyes ecuatorianas para obtener beneficios tributarios, en este caso, se declaran sin fines de lucro y son reguladas por la Superintendencia de Economía Popular y Solidaria. Para estos casos la ley explica:

De acuerdo al presente Reglamento se entiende por organización sin fines de lucro, aquella cuyo fin no es la obtención de un beneficio económico sino principalmente lograr una finalidad social, altruista, humanitaria, artística, comunitaria, cultural, deportiva y/o ambiental, entre otras. En el caso de que su actividad genere un excedente económico, éste se reinvertirá en la consecución de los objetivos sociales, el desarrollo de la organización o como reserva para ser usada en el próximo ejercicio. (Decreto $\mathrm{N}^{\circ} 193$, 2017, art. 3)

Es necesario explicar este contexto porque es en las asociaciones donde se practica la asociatividad, concebida como estrategia para el desarrollo económico.

Indicadores de la asociatividad: para la medición de la asociatividad, se lo realiza a través de cómo practican los socios la estrategia de asociarse, de igual manera, cómo perciben el desempeño de la dirigencia y de los otros socios. Los indicadores que convienen a esta población de estudio son: pertenencia (sentido de pertenencia), liderazgo empresarial, competencias del socio y cooperación del socio. Cada indicador tiene sus preguntas claves para obtener información. Hay que recalcar, que en cada uno de estos de estos indicadores está la percepción de confianza, más aún en las competencias del socio, pues, en la producción de cacao es fundamental la confianza, el daño que puede causar si un socio trabaja con un plaguicida no permitido en ocasiones es irrecuperable, se culpa a todo el lote, la asociación pierde prestigio.

Desarrollo económico: una corriente de pensamiento de la economía trata el tema solo como crecimiento económico, y define al mismo como: "La expansión de las posibilidades de producción" (Parkin, 2014, p. 38). Por otro lado, existen críticas sobre esta forma de apreciar el concepto, según Sachs (2015), "Los economistas acostumbran a resumir el nivel de desarrollo de una economía con el producto interno bruto (PIB) por persona" (p. 33). Para Sachs con esta forma de medición, se observa solo a nivel país, pero no lo que pasa individualmente, así, habrá personas que sean favorecidos y otros no lo serán con el crecimiento económico. En el caso del sector cacaotero, las cifras macroeconómicas son interesantes, las exportaciones de cacao del Ecuador en el año 2017 alcanzaron las 290000 toneladas métricas, midiendo como punto de referencia, que en el 2003 se produjo 100000 (Ministerio de Agricultura y Ganadería, 2017). Ha aumentado las posibilidades de producción y los ingresos han sido muy buenos. Conviene conocer si existe una distribución equitativa de la riqueza. Por esta razón, conviene definir desarrollo económico como el bienestar que produce el crecimiento económico en los habitantes de un país.

Aunque es un tema con muchas perspectivas, se puede definir desarrollo económico de una persona, como la búsqueda constante de aumentar el bienestar propio a través de un trabajo eficaz. Como se aprecia, es un concepto que atañe a lo personal más no a lo colectivo. Por lo general, este término se lo utiliza a nivel macroeconómico, como la capacidad de un estado de proporcionar el bienestar a sus habitantes. Sin embargo, también se lo puede aterrizar a nivel personal, por efecto de la propiedad de la relación de inclusión entre conjuntos. Es fundamental que exista un desarrollo gradual en la economía de las personas en general, el productor de cacao necesita también, que sus inversiones le produzcan bienestar, mejore su calidad de vida, o por lo menos solucione sus problemas para satisfacer sus necesidades básicas. La producción de cacao debe redituar lo suficiente para el logro de estos propósitos.

Indicadores de desarrollo económico: a nivel macroeconómico, se puede utilizar los indicadores dados por los centros de investigaciones del país para medir el desarrollo. Sin embargo, para esta investigación es conveniente utilizar indicadores para la variable desarrollo económico, que muestren la conformidad del beneficio dado por asociarse, además, de mejoras, aunque sean pequeñas, en beneficios logrados. Entre estos indicadores se tiene:, entrega de insumos, tiempos de cosecha, compradores, áreas cultivadas, rendimientos por hectárea, beneficios en transportes e incrementos de la producción. 


\section{Metodología}

La investigación es de tipo cuantitativa, aplicada, no experimental y transeccional correlacional. Las variables a medir la relación son asociatividad y desarrollo económico. La unidad de análisis es el agricultor que produce cacao y que pertenece a una asociación agrícola. La población de estudio son los agricultores agremiados a las asociaciones o corporaciones de primer grado que tienen como actividad principal la comercialización de cacao. El territorio delimitado fue la provincia de Los Ríos. Según el boletín situacional del cacao, al año 2016, están censados 2538 productores de cacao asociados a una corporación (Ganadería, 2016). Es de suponerse que continuamente se incorporan más agricultores y así mismo salen de las asociaciones. Pero, el dato es suficiente para calcular una muestra de una población finita. Se obtiene una muestra de 334 personas a ser encuestadas. El instrumento de recolección de datos es la encuesta. Se realizó una muestra por conglomerados, para que sea representativa a la población. Se escogió dos asociaciones por cada cantón perteneciente a la provincia (13 cantones en total); posteriormente, se hizo contactos con los presidentes de las asociaciones para convenir fechas en las cuales se podía hacer la encuesta, se realizó entre 12 y 13 encuestas por asociación.

En la figura 1 se esquematiza como fueron tratadas las variables (operacionalización), es decir, los indicadores que suman a la variable asociatividad (considerada como variable independiente) y los indicadores que suman a la variable desarrollo económico (considerada como variable dependiente).

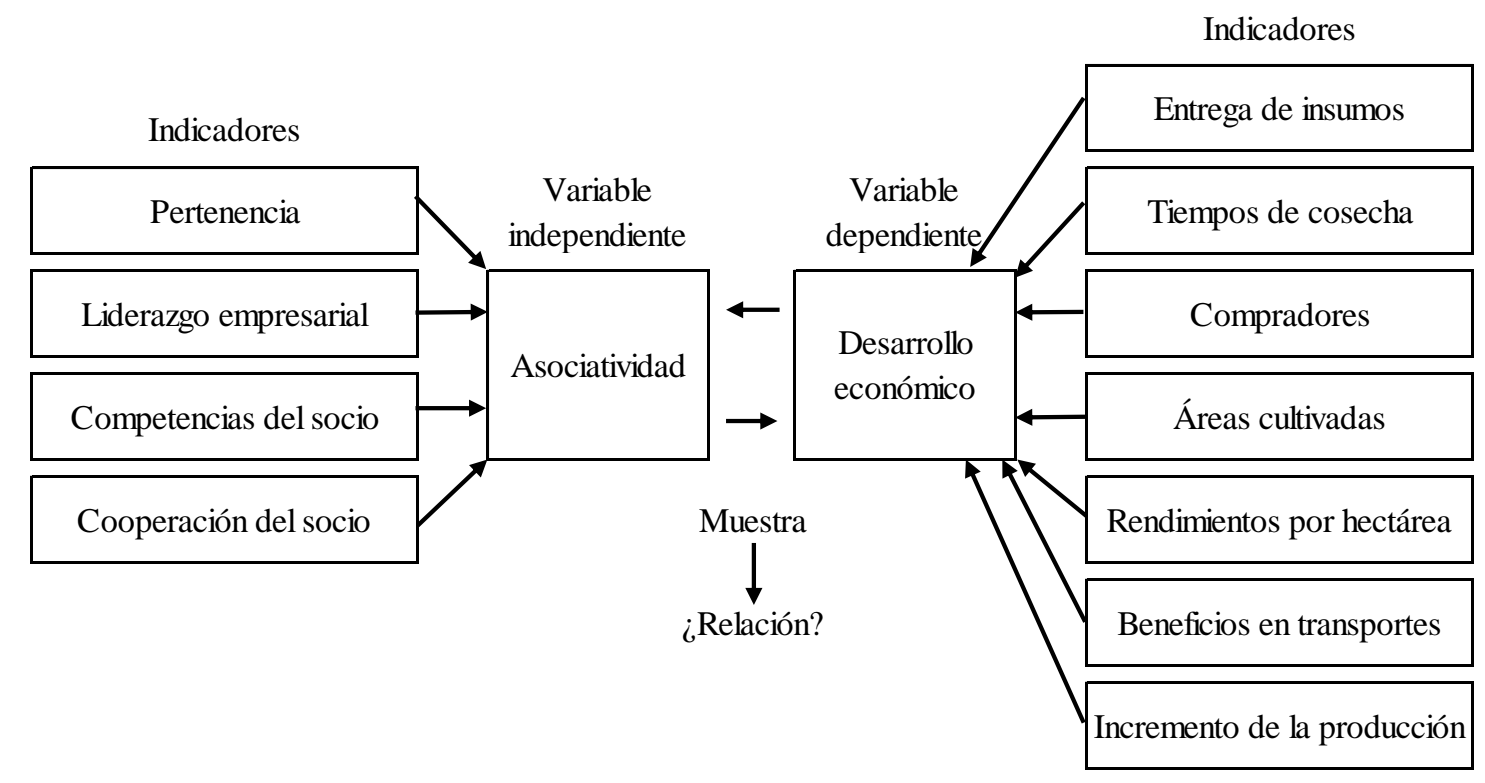

Figura 1. Esquema de la operacionalización de las variables

Fuente: Autor

Para cumplir con el objetivo de la investigación, se planteó y contrastó estadísticamente la siguiente hipótesis: La asociatividad influye significativamente en el desarrollo económico de los productores de cacao en la provincia de Los Ríos del Ecuador.

Para el contraste de la hipótesis, se utilizó las pruebas de independencia chí cuadrado con programa SPSS versión 21, se calculó mediante el estadístico exacto de Fischer, porque en la tabla de contingencia se presentaban frecuencias esperadas menores a cinco. Para aceptar la hipótesis de la investigación se lo realiza por el valor $\mathrm{p}$, que debe ser menor o igual a 0,05 . Adicionalmente, se obtuvo el coeficiente rho de Spearman, para medir el grado de correlación. El baremo para interpretar la correlación rho de Spearman es: bajo, de 0,00 a 0,29; moderado, de 0,30 a 0,69; alto, de 0,70 a 1,00; sean positivos o negativos (Castejón, 2011). A través de contrastar la hipótesis, se logra cumplir con el objetivo de la investigación, puesto que, se demuestra si existe o no relación o influencia entre las variables y si es significativa.

\section{Resultados y discusión}

Se plantea las siguientes hipótesis:

Hipótesis nula (H0): La asociatividad no influye significativamente en el desarrollo económico de los productores de cacao en la provincia de Los Ríos del Ecuador.

Hipótesis alternativa (H1) o hipótesis de la investigación: La asociatividad influye significativamente en el desarrollo económico de los productores de cacao en la provincia de Los Ríos del Ecuador.

Se obtiene lo siguiente: 
Tabla 1 Tabla de contingencia

\begin{tabular}{|c|c|c|c|c|c|c|c|c|}
\hline & & & \multicolumn{5}{|c|}{ Desarrollo económico } & \multirow{2}{*}{ Total } \\
\hline & & & Nunca & $\begin{array}{c}\text { La } \\
\text { mayoría } \\
\text { de veces } \\
\text { no }\end{array}$ & $\begin{array}{l}\text { Algunas } \\
\text { veces sí, } \\
\text { algunas } \\
\text { veces no }\end{array}$ & $\begin{array}{c}\text { La } \\
\text { mayoría } \\
\text { de veces } \\
\text { sí }\end{array}$ & Siempre & \\
\hline \multirow{5}{*}{ Asociatividad } & \multirow{2}{*}{$\begin{array}{l}\text { Algunas veces sí, } \\
\text { algunas veces no }\end{array}$} & Recuento & 3 & 10 & 11 & 5 & 1 & 30 \\
\hline & & Frecuencia esperada & 0,3 & 1,4 & 13,7 & 13,1 & 1,4 & 30 \\
\hline & $\begin{array}{l}\text { La mayoría de } \\
\text { veces sí }\end{array}$ & Recuento & 0 & 6 & 138 & 131 & 6 & 281 \\
\hline & \multirow{2}{*}{ Siempre } & Recuento & 0 & 0 & 4 & 10 & 9 & 23 \\
\hline & & Frecuencia esperada & 0,2 & 1,1 & 10,5 & 10,1 & 1,1 & 23 \\
\hline \multirow{2}{*}{ Total } & & Recuento & 3 & 16 & 153 & 146 & 16 & 334 \\
\hline & & Frecuencia esperada & 3 & 16 & 153 & 146 & 16 & 334 \\
\hline
\end{tabular}

Fuente: Datos procesados en SPSS, tomados del estudio de campo

Interpretación: en la tabla 1, se presenta una tabla de contingencia con 8 grados de libertad, se muestra que existen 7 casillas con frecuencias esperadas menor a 5 . Se utilizó la misma escala de Likert utilizada en las encuestas para recodificar la sumatoria de los datos de cada variable para luego transformarlas en las variables asociatividad y desarrollo sostenible que mantienen la misma escala de valores. Con estas variables recodificadas se estableció la tabla de contingencia.

Tabla 2 Pruebas de chi-cuadrado

\begin{tabular}{lrrrr}
\hline & Valor & gl & $\begin{array}{c}\text { Sig. } \\
\text { asintótica } \\
\text { (bilateral) }\end{array}$ & $\begin{array}{c}\text { Sig. exacta } \\
\text { (bilateral) }\end{array}$ \\
\hline Chi-cuadrado de Pearson & $158,378 \mathrm{a}$ & 8 & 0 & 0 \\
Razón de verosimilitudes & 84,627 & 8 & 0 & 0 \\
Estadístico exacto de Fisher & $\mathbf{8 2 , 4 3 9}$ & & & $\mathbf{0}$ \\
Asociación lineal por lineal & $61,870 \mathrm{~b}$ & 1 & 0 & 0 \\
N de casos válidos & 334 & & & \\
\hline
\end{tabular}

a. 7 casillas $(46,7 \%)$ tienen una frecuencia esperada inferior a 5. La frecuencia mínima esperada es 0,21. b. El estadístico tipificado es 7,866.

Fuente: Datos procesados en SPSS, tomados del estudio de campo.

Interpretación: en la tabla 2, se toma como referencia el estadístico exacto de Fischer para obtener la significancia bilateral (valor p), en este caso se obtuvo 0,000. La razón por la que se toma o se calcula con el exacto de Fisher es porque se tiene más del $20 \%$ de casillas con una frecuencia esperada inferior a cinco (Soporte de Minitab, 2018).

Tabla 3 Pruebas de chi-cuadrado

\begin{tabular}{|c|c|c|c|c|}
\hline & & & Asociatividad & $\begin{array}{l}\text { Desarrollo } \\
\text { económico }\end{array}$ \\
\hline \multirow{6}{*}{ Rho de Spearman } & \multirow{3}{*}{ Asociatividad } & Coeficiente de correlación & 1 &, $364 * *$ \\
\hline & & Sig. (bilateral) & . & 0 \\
\hline & & $\mathrm{N}$ & 334 & 334 \\
\hline & \multirow{3}{*}{ Desarrollo económico } & Coeficiente de correlación &, $364 * *$ & 1 \\
\hline & & Sig. (bilateral) & 0 & . \\
\hline & & $\mathrm{N}$ & 334 & 334 \\
\hline
\end{tabular}

**. La correlación es significativa al nivel 0,01 (bilateral).

Fuente: Datos procesados en SPSS, tomados del estudio de campo.

Interpretación: en la tabla 3, se obtiene el grado de correlación rho de Spearman, que es igual a 0,364, que conforme al baremo de interpretación, se tiene una correlación positiva moderada. Además, la significancia es de 0,000; se puede decir, que existe poco riesgo de concluir que existe correlación. 
Resultados: como el valor del nivel de significancia (exacto de Fisher) es de 0,000 y es menor a 0,05 , se rechaza la hipótesis nula (H0) y se acepta la hipótesis alternativa (H1) o hipótesis de la investigación. Se puede indicar que las variables tienen una asociación estadísticamente significativa. Por tanto, se expresa que la asociatividad influye significativamente en el desarrollo económico de los productores de cacao en la provincia de Los Ríos. Además, que el grado de correlación Rho de Spearman es de 0,364, es decir, que las variables tienen una correlación positiva moderada.

Discusión: cuando se mide percepciones a través de la escala de Likert es conveniente utilizar las pruebas chicuadrado o pruebas de independencia, son las adecuadas para medir datos de variables ordinales y categóricas, sin embargo, hay una duda porque no se demuestra que tan dependientes son las variables, solamente se contrasta que no se puede negar que hay una asociación entre las variables. Por esta razón, se usó el estadístico rho de Spearman para observar el grado de correlación. Con los datos obtenidos se determina una relación o influencia, pero no se puede determinar concluyentemente una causalidad, esto es, que la asociatividad cause el desarrollo económico. Hay muchos factores o variables intervinientes que provocan un auge o declive en el desarrollo económico como son la estabilidad política, social, la buena gobernanza, los fenómenos climáticos, entre otros; la sequía o lluvias intensas harían colapsar las economías de los agricultores. Otro punto de vista, es que existe un impedimento grande de buscar causalidad entre estas dos variables, está dado por la dificultad de hacer un experimento social. Para realizar un experimento, se tendría que observar a través de varios años las ventajas económicas de haberse asociado y realizar un análisis comparativo relativo con otros que nunca se asociaron; también, se puede hacer dos grupos, uno de control, quienes no se les permitirá asociarse y a otro que sí. Los resultados se tendrían después de un año. Lo dificil será conseguir grupos homogéneos voluntarios de agricultores y decirles que unos no se asocien y a otros que sí.

\section{Conclusiones}

Con respecto al objetivo de la investigación, se concluye que la asociatividad influye significativamente en el desarrollo económico de los productores de cacao en la provincia de Los Ríos del Ecuador.

Se concluye de manera general, que la asociatividad contribuye a incrementar los ingresos en dos sentidos, mejorando los precios y disminuyendo los costos de producción. Dicho de otra manera, por un lado, en las ventas con mejores precios, porque se obtiene poder de negociación al ofertar grandes cantidades de manera frecuente y con confianza de que es un buen producto. Por otro lado, el conocimiento adquirido al estar asociado (aprendizaje colaborativo) hace que los agricultores sean más eficientes con sus costos de producción, es decir, si menos cuesta producir y si se mantienen los precios de venta, entonces, mayor será la utilidad.

\section{Referencias Bibliográficas}

Asamblea Nacional. (2016). Ley Orgánica de Tierras Rurales y Territorios Ancestrales. Quito, Ecuador.

Borja, C., Alarcón, G., y Quizhpe, V. (2017). "La asociatividad de las organizaciones sociales del sector subtrópico de la provincia Bolivar". En linea. Revista Observatorio de la Economía Latinoamericana".

Brassel, F., Herrera, S., y Laforge, M. (Eds.). (2008). ¿Reforma agraria en el Ecuador? : viejos temas, nuevos argumentos. Quito: SIPAE.

Castejón, O. (2011). Diseño y análisis de experimentos con Statistix. Maracaibo, Venezuela: Universidad Rafael Urdaneta, Fondo Editorial Biblioteca.

Cámara de Comercio de Bogotá. (2010). Asociatividad: Estrategia para la internacionalización de su negocio.

Coordinadora Ecuatoriana de Comercio Justo. (2018). ¿Qué es Fairtrade? Descargado de https : / / goo - $91 /$ hgqt $2 j$

Decreto No193. (2017). Reglamento para el Otorgamiento de Personalidad Jurídica a las Organizaciones Sociales. Quito, Ecuador: Registro Oficial, Órgano del Gobierno del Ecuador.

Ganadería, M. d. A. y. (2016). Boletín situacional cacao. Quito, Ecuador.

Ministerio de Agricultura y Ganadería. (2017). Producción de cacao. Descargado de https://goo.gl/ ne 7 F $7 Y$

Parkin, M. (2014). Economía. México: Pearson Educación de México.

Programa de las Naciones Unidas para el Desarrollo. (2000). Desarrollo Humano en Chile 2000 (Inf. Téc.). Santiago de Chile.

Sachs, J., y Vilà, R. (2015). La era del desarrollo sostenible. Barcelona, España: Deusto.

Soporte de Minitab. (2018). Pruebas chi-cuadrado. Descargado de https://goo.gl/auzcnh

Recibido: 30 de junio de 2018

Aceptado: 3 de septiembre de 2018 
